\title{
Organization of a Tumor Bank: The Experience of the National Cancer Institute of Mexico
}

\author{
L. Ruíz-Godoy A. Meneses-García L. Suárez-Roa V. Enriquez \\ R. Lechuga-Rojas E. Reyes-Lira
}

National Cancer Institute of Mexico (INCan), Mexico City, Mexico

\section{Key Words}

Tumor bank $\cdot$ Tissue banking $\cdot$ Quality control

\begin{abstract}
A tumor bank (TB) is an ordered collection of neoplastic samples, normal tissue, and/or fluids preserved under optimal conditions, as well as storing patients' clinical information. The objective of this article is to outline the planning and logistics necessary for the functioning of the Instituto Nacional de Cancerología (INCan) TB in Mexico City. For the planning and logistics of a TB, several technical, legal, medical, structural, and physical aspects were considered, which can be grouped under four headings: (1) design and structure, (2) equipping the area and informatics, (3) ethical-legal aspects, and (4) sample collection, preservation, and quality control. One crucial element of interinstitutional interest will be the transfer of these concepts to the different oncological centers, integrating in this manner a network that enables the exploration of the different pathologies from therapeutic, epidemiological, and molecular points of view.
\end{abstract}

Copyright $\odot 2010$ S. Karger AG, Basel (c) 2010 S. Karger AG, Basel

$1015-2008 / 10 / 0773-0147 \$ 26.00 / 0$

Fax +4161306 1234

E-Mail karger@karger.ch

www.karger.com
Accessible online at: www.karger.com/pat

\section{Introduction}

A tumor bank (TB) includes an ordered collection of neoplastic samples, normal tissue, and/or fluids preserved under optimal conditions, as well as the storing of patients' clinical information. The samples collected and preserved in TBs are used for translational, basic, and clinical research $[1,2]$. The collection of tumor tissues employed for cancer research is not new; historically, tumor samples were obtained from pathology files in which samples for diagnosis had been maintained in paraffin blocks for indefinite time periods. Current knowledge in biomedicine, mainly in the molecular characterization of cancer, makes it necessary to develop TBs. The analysis of fresh tissue and clinical information relevant to investigations in which the patient is not directly involved gives rise to questions about the ethical and legal viewpoint $[3,4]$. The installation of a TB should be a multidisciplinary development with collegiate and consensual decision-making. The challenge of ethics and the balance between clinical practice and research play a relevant role in the clinical and medical fields.

The contribution of the TB is relevant at different levels. Preserving multiple samples of the same tumor type

Dr. L. Ruíz-Godoy

Banco de Tumores, Instituto Nacional de Cancerología

Av. San Fernando 22

Tlálpan, 14080 México, DF (Mexico)

Tel. +52 555628 0400, ext. 278, Fax +52 555573 4664, E-Mail lruizgodoy@gmail.com 
with integrity of their cellular components and the relevant clinical information makes the TB a valuable vehicle for carrying out molecular studies at different cellular levels, for the study of carcinogenesis, the biological behavior of the tumor, response and resistance to drugs, etc. There are many achievements in the short, medium, and long term, and the aim is improvement of the patients' health.

The need for agile translation into clinical practice and the knowledge generated by basic research have given rise to translational medicine. It is generally noteworthy that frequently basic research is forgotten or does not make a useful contribution to the treatment of the patient. Certainly in developing countries, the knowledge acquired over the past 3 or 4 decades has not experienced adequate parallelism in the repercussion on the management of the patient. Today, we cannot postpone the importance of the activity of translational medicine, because it is a multidisciplinary responsibility and not exclusively the concern of those involved in clinical practice, in which the TB plays a fundamental role. Interinstitutional, national, and international collaborations can be made from TBs, whether from a sole tumor type, from diverse tumors, or even from pre- and posttreatment, in any of their modalities, which can comprise head and neck, breast, or pancreatic tumors [5-7].

It is not known exactly which TB was the first to operate regularly; however, in recent years extensive TB networks have been formed that operate in a regular manner, such as the TuBaFrost Consortium in Europe [8], the Cooperative Human Tissue Network [9] in the US formed in 1987, and the Spanish National Cancer Research Centre-Spanish National Tumour Bank Network (CNIOSNTBN) [10], among others, all of which contribute to the globalization of science.

The objective of this article is to outline the planning and logistics that were carried out for the functioning of the Instituto Nacional de Cancerología (INCan) TB in Mexico City.

\section{Tumor Banks}

For the planning and logistics of a TB, several technical, legal, medical, structural, and physical aspects were considered, which can be grouped under four headings: (1) design and structure, (2) equipping the area and informatics, (3) ethical-legal aspects, and (4) sample collection, preservation, and quality control.

\section{Design and Structure}

\section{Design}

The infrastructure was planned under the Official Mexican Norm NOM-166-SSA1-1997 for the organization and functioning of clinical laboratories. The physical area was chosen based on availability in form, size, and localization in proximity to surgical areas. A contiguous and independent area was adapted for the administrative office.

In the architectonic program, requirements were established for areas that make up the $\mathrm{TB}$, analyzing the spatial and organizational structure, and complying with the objectives and functions which were described.

\section{Infrastructure}

The physical structure was designed to incorporate all necessary modifications, such as sanitary floor and wall finishes, and the supply of water, $\mathrm{CO}_{2}$, and $\mathrm{NL}_{2}$ pipelines.

The electrical wiring and the installation of sockets had to comply with established norms and specifications. The air conditioning employs a double high-efficiency particle arresting (HEPA) filter. HEPA filters are capable of removing the majority of airborne hazardous particles, including mold spores, dust, dust mites, and other irritating allergens. They retain approximately $99.97 \%$ of particles with a size of up to $0.3 \mu \mathrm{m}$ [11], which helps maintain the air clean in the TB and sterility in the processes that are performed.

\section{Furnishings}

The architectonic project was developed using furnishings, equipment, installations, and construction specifications according to planning and compliance with Mexican national sanitary norms [12]. The furnishings installed are made of aluminum with a granitetopped table for the analytical scale.

\section{Area Equipment and Informatics}

\section{Software}

There is some commercial software for clinical databases, such as that developed by the Data Corp. (Houston, Tex., USA). This database was developed at Microsoft Visual FoxPro (Redmond, Wash., USA). This software runs on a Windows Professional 2000 operative system and was designed for pancreatic TBs [7]. Other TBs have noncommercial software, which is designed according to 
specific user needs, such as the S. João de Portugal Hospital TB software [13, 14].

INCan TB software was designed according to the needs of the TB and of safe keeping the identification of the patient; for access to the software, several access keys were designed for the user, technical personnel, area coordinator, or researcher. Only the general and the area coordinators have access to all the information, and the areas to which personnel have software access depend upon their functions. An interface was generated with the electronic clinical file of the institute (called INCaNet), which continuously updates all clinical information of patients. The first step for access to the software involves scanning the signed letter of informed consent.

For access to information of the patient, it is indispensable that the informed consent letter be there; the patient is registered, and later, the sample is saved. To save or remove a sample from the ultrafreezer, we designed a layout that provides ease of tissue localization and one which at the same time facilitates sample management, in addition to possessing all of the sample collection information such as date, time, localization, time of freezing, and localization in the ultrafreezer. In addition, the protocol to which the tissue was assigned is indicated.

Software for the TB was designed to include different sections, such as quality control, documents, and information tags. There is also a system in which variables can be crossed and in which search limits can be established, such as tumor type, patient gender, treatments, tumor recurrences, etc., with which groups of specific samples that are required can be selected.

Each time the system is accessed, registry of the access key used, date and time of access, the activity carried out, and the time of exit are recorded. The TB software has a link with the pathology software, as well as with that of the blood bank.

\section{Equipment}

Equipment can be divided into equipment for general use, equipment for sample preservation, and equipment for quality control. Equipment for general use comprises all equipment that is required in a laboratory for making solutions, sterilization equipment, storing reagents, etc., as well as its parts: an autoclave, an analytical scale, a pH measurement device, a centrifuge, refrigerators, and a spectrophotometer. In the preservation area, we have a laminar flow dome, equipment for sample slicing, freezing equipment with ramps, an ultrafreezer set at $-80^{\circ} \mathrm{C}$ with $\mathrm{CO}_{2}$ backup and a telephone alarm, $\mathrm{NL}_{2}$ tanks, and an oxygen sensor. Equipment used for quality control in-

Organization of a Tumor Bank cludes a thermocycler, an infrared beam detection system, electrophoresis chambers, power sources, a transfer chamber, and some lesser equipment.

\section{Ethical-Legal Aspects}

\section{Patient Privacy}

Anonymity is the state of a person when his or her identity is unknown. Different circumstances can make anonymity necessary, and here there are legitimate reasons such as the privacy of the patient. However, the term anonymity is employed in the following different guidelines:

(a) It can be applied, as in the US, as anonymized (not identifiable), i.e., no one can identify the patient, no one knows the patient's follow-up once the tissue sample has been acquired.

(b) Another type of privacy is which information is identifiable, but this information can only be accessed by a code [15].

Some biobanks (BBs) or TBs used a double code, which renders patients identifiable [16]. The patient already has a code, and in the TB he/she is given another code, so that only authorized personnel have access to the information. The researcher who asks for samples from the TB is only given the information necessary for the study that he/she is about to do. In the INCan TB, we employ this latter type of privacy.

For biomedical research and translational medicine, anonymous tissue samples for which there is no access to the patient's clinical information; they have very limited use, because it is impossible to obtain results that are conclusive and applicable to the patient for improvement in treatment and/or quality of life [17].

\section{Letter of Informed Consent}

The utilization and cession of human samples for research and hence the existence of BBs and TBs are based on four general principles that are a constant in the law of biomedical investigation and that seek to afford guarantees to the patients and to the researchers themselves: (a) express and specific informed consent of the patients as an indispensable element for the research, (b) scientific justification and adaptation of the research through the presentation of approved and financed projects, (c) gratuity for the samples and equal opportunities in accessing samples, and (d) protection of personal data.

These essential ethical limitations are those that are generally accepted by international agreements and that 
follow the European Commission: Ethical, Legal and Social Implications of Genetic Testing, 2004 [16]. Similarly, all BBs should be assigned to an ethical and research committee, whose members should conduct surveillance for the correct functioning and adaptation of $\mathrm{BB}$ and $\mathrm{TB}$ procedures regarding these ethical principles.

In Mexico, although it is not expressed with the term 'informed consent', the concept can be found in legal documents and legal norms of informed consent of 2 decades ago: in the General Health Law published in the 'Diario Oficial de la Federación' in 1984. Later, when this concept was defined as informed consent, it was included in Article 14, Fraction V of the Regulations of the General Health Law in Matters of Research for Health, which was published in Mexico in 1987. Since 1999, with the publication of the official Mexican norm for clinical records, these authorization and consent forms are redefined when new medical acts are added, such as transplants, clinical investigation in humans, hospital necropsy, and procedures involving mutilation. However, the donation of tissues to a TB for research is not specified.

The Italian Biobank User Group (IBUG) reviewed 20 different organizations in different countries, comparing their laws, norms, and regulations, as well as carrying out an investigation of several informed consent forms from protocols conducted at three institutions. In the study, the group observed four different informed-consent models as follows: ample consent, partially restricted consent, several consent plans, and specific informed consent. Based on this analysis, IBUG members proposed unified informed consent [17].

Unified informed consent refers principally to the property of the biological material derived from surgical interventions, the potential use of the biological material, the patient's clinical information, confidentiality and later commercial data, such as diagnostic procedures and drugs, and intellectual property (IP) rights such as patents and licenses [18].

Informed consent as applied at the INCan TB was conducted in a consensual manner by some members of the institute's Bioethics Committee and the TB. This is somewhat similar to the study of Salvaterra et al. [19], and informed consent was validated by the Bioethics Committee.

The debate continues in the international arena at different levels with respect to permission with generalized consent, whether or not to request consent each time the tissues are employed in a research protocol, and what the information should contain, such as the fact that the laws and norms of each country are different is somewhat complex in terms of unifying informed consent [16-21]. Thus, it is recommended to adhere to the norm in each country and institution.

Another issue that is subject to discussion is the authorization to administer informed consent as a postoperative process, because sample utilization could occur even when informed consent has not been granted. However, if access to samples is administered by a third party, that is, a BB or a TB, which works as a legitimate and ethical intermediary complying with approved protocols, this no longer constitutes a problem. BBs and TBs are being converted into a recognized discipline with distinct knowledge, techniques, and national policy guidelines in several countries and international associations. The advent of TBs also helps in safeguarding the patient's confidentiality. The intermediary collects, designs a second code, and provides clinical data to researchers in such a way that the data will be impossible to identify, either directly or indirectly, by third parties.

Informed consent is customarily provided prior to any procedure; however, in the case of donation of excessive tissue, in practice it would be very useful to be able to obtain agreement from the patient postoperatively. Some ethical committees have demonstrated reluctance in approving the postoperative consent process. According to Hewitt et al. [22], there is a great advantage in obtaining postoperative consent, and the authors find no reasonable objection for the use of surgical tissues and specimens associated with medical data, when this is duly regulated. They propose that if the consent decision is not obtained (not conceded or denied) within a moratorium period of 1-3 months, the samples can be denominated as anonymous for research, bearing local legislation in mind. If consent is denied, the samples should be destroyed if they are not necessary for diagnostic ends. In many cases, postoperative consent is not only more practical, but also, under certain circumstances, could in reality be more ethical. One virtue of accepting a time period for finalizing the consent process with defined limits that include the pre- as well as the postoperative period is that this creates the opportunity for analysis and flexibility in decision-making by patients who wish to donate their tissues. In general, this regulation requires the services of a $\mathrm{BB}$ to guarantee that the processes are carried out, documented, and supervised independently and impartially. The result of this will afford improvement in the acceptance of the public, their participation, and the availability of tissues. 
In our experience at the INCan TB, obtaining postoperative informed consent would be of great help in the daily practice of sample collection, as many samples cannot be acquired due to a lack of consent prior to the patient's appointment. Our group is in favor of the proposal of the group of Hewitt et al. [22].

\section{Intellectual Property}

The use of human tissues obtained during the course of diagnosis or treatment and to be preserved and used in future research protocols is a complex theme that remains unsolved from social, ethical, and legal standpoints. The situation of IP requires discussion among regional institutions, organizations, and networks, as well as recognition of and compliance with existing agreements. The CAPCA Tumor Bank Working Group examined the possible models under study or those that have been established by groups such as the Online Community Research Network (OCRN) and the British Columbia Cancer Agency-Tumour Tissue Repository (BCCATTR) [23].

The theme of IP and exclusive rights to biological material donated by patients is one that provokes various questions, such as, 'Who should decide when there is a limited amount of material?', and 'Who should have access to patients' data and samples?' [24].

The formation of TBs is developed based on donations of biological material in a spirit of solidarity; the material should not be monopolized by a small group of researchers. As the number of BBs with diverse characteristics, tissue samples, DNA, blood, fluids, etc., increases, the perspective has changed [23]. Frequently, TBs and BBs are not well documented, which leads to resistance and lack of the definition of responsibility, rendering the researchers' practice difficult [24].

'Many academic scientists express confusion, if not consternation, concerning the management and practical implications of possession, custody, database reliability, and IP in general' [23]. In the experience of this organization within the INCan, a similar situation occurred; however, keeping the staff informed and involved for the benefit of generating research with tumor tissue preserved under freezing conditions has mitigated this reaction little by little.

\section{Recuperation Costs}

In daily practice, there are great uncertainties with regard to the main norms established and the security of the transparent management of financial, economic, and biological resources. This is a normal change, depending on

Organization of a Tumor Bank the project's financing, that is, private, public, or semiprivate. The ambiguity that exists regarding the state of the genetic material makes it necessary to consider several points, such as property, rights, remuneration or compensation of the participants, financial earnings, beneficence or welfare, and other types of earnings [24, 25].

Tissue or tumor banks should be noncommercial. In Europe, researchers do not rule out the possibility of industry exploiting the results of investigations carried out on human tissue samples. In cases in which the academic and commercial worlds collaborate in research, the academic results should be distributed freely for the public good, so as to avoid possible conflicts and even 'conflicts of interest' [26].

It has been proposed that donors should benefit economically if their tissues are used commercially. This is a difficult question to answer. It is not acceptable in Europe [27]; however, in the US, there are strong opinions in favor of financial incentives for donors [28].

At the INCan, the Tumour Bank Committee makes the decisions on the protocols that are accepted for supplying biological samples; these protocols need to have been previously accepted by the bioethical and scientific committees.

The cost of the process of sample preservation in the case of the internal INCan protocols is absorbed by the institute itself; however, because the TB at the INCan has been created recently, a recuperation quota should be considered for external protocols, including those of the pharmaceutical industry.

\section{Sample Collection, Preservation, and Quality Control}

The sample collection was planned in line with the established processes for diagnosis and surgical treatment of patients; however, this was difficult and the initial flow chart was amended on several occasions (fig. 1).

\section{Sample Collection}

Prior to the opening of the TB, and based on the tumors that are most frequently received at the INCan, the TB Committee made the decision to collect the following tissues: breast cancer, cervicouterine cancer, ovarian cancer, lung cancer, colorectal cancer, renal cancer, prostate cancer, head and neck cancer, and prostate cancer in addition to tissues processed according to protocols approved by the INCan Bioethical and Research Committees.

As at the S. João de Portugal Hospital TB [10], one of the principal operative problems that we have encoun- 
Fig. 1. Algorithm of the processes carried

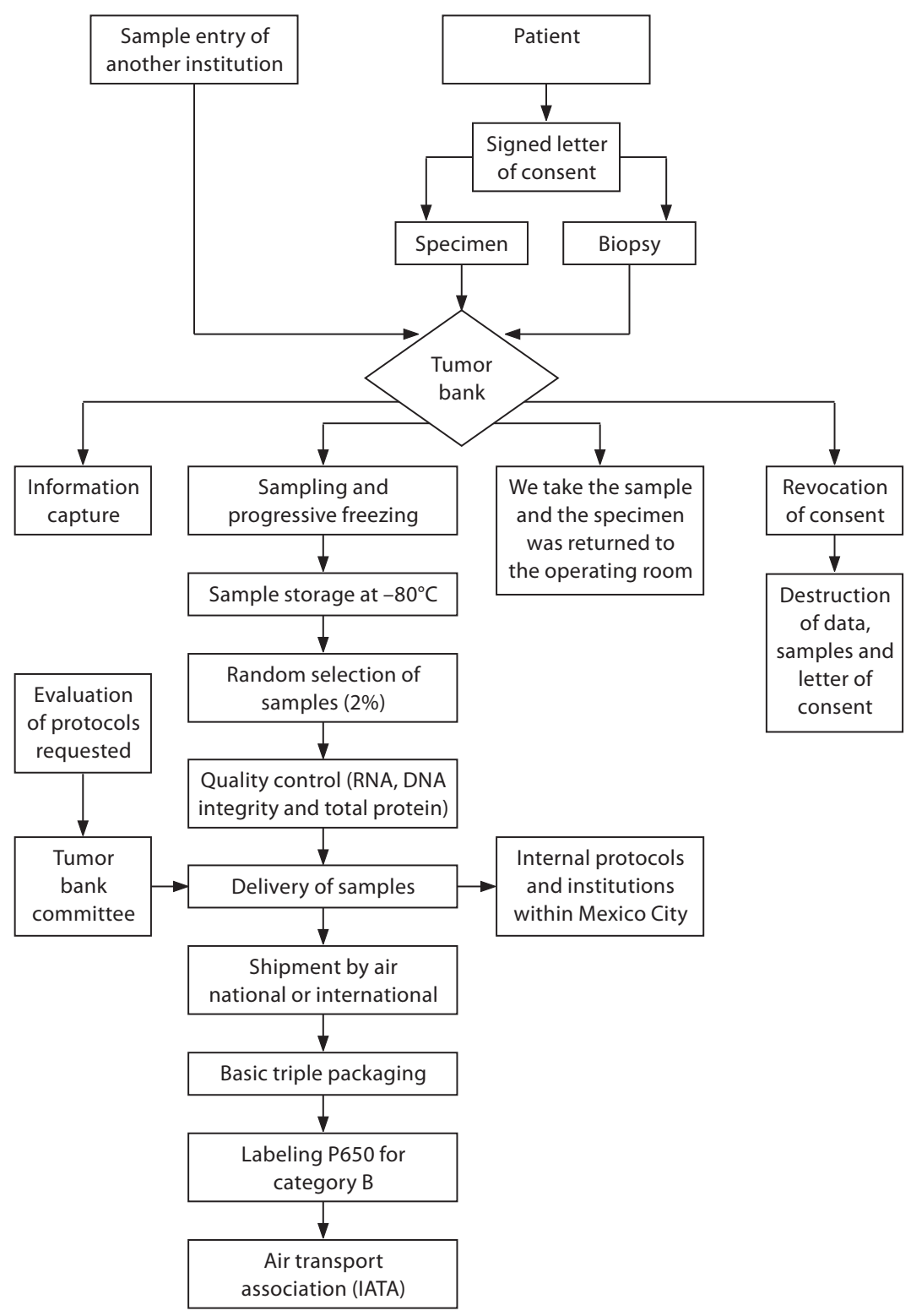
out at the INCan TB.

tered at the INCan TB involves human resources and staff training. Collaboration of staff including physicians and nurses who work in the different areas was scarce. Likewise, it was necessary to train the laboratory staff who participate in the TB.

\section{Biopsies}

The biopsies collected at the TB are from breast, cervicouterine, and prostate tumors, as well as small biopsies (by means of Tru-cut). For fresh tissue control, an imprint is performed, which is stained with $\mathrm{H} \& \mathrm{E}$.

\section{Surgical Specimens}

The surgical specimen is transported immediately after extirpation to the TB in a biosafety dome. It is then dissected by a pathologist, making sure that it does not have ischemic or necrotic zones. Depending on the tumor, the pathologist decides on the slices necessary, so as 
not to interfere with the correct diagnostic-histopathological process. Similarly, a mirror cut is performed as a histological control; this is embedded in paraffin and stained with $\mathrm{H} \& \mathrm{E}$.

\section{Preservation}

Samples, biopsies, as well as surgical specimens are frozen in cryovials by means of an $\mathrm{NL}_{2}$ vapor recovery system with ramps; these are stored in an ultrafreezer at $-80^{\circ} \mathrm{C}$. This equipment has safety measures, including a triple visual, acoustic, and remote alarm with emergency telephone calls; therefore, if the temperature increases, the $\mathrm{CO}_{2}$ backup injection system is activated, which makes it possible to transport the samples to another refrigerator.

\section{Quality Control}

Quality control is performed monthly in 2\% of the frozen samples. RNA, DNA, and protein extraction are conducted on the samples, which are randomly select; likewise, immunohistochemistry is conducted in histological control blocks for protein expression.

\section{Histopathology}

The fixed tissue is assessed to evaluate the mirror-cut tissue sampled in the case of surgical specimens and the imprints in the case of biopsies. Immunohistochemistry is performed to evaluate protein expression with the following antibodies: vimentin, CD34, and Bcl-2.

\section{Genomic Quality Control}

Evaluation of molecular integrity is performed by means of the DNA and RNA column-based extraction, using the MicroRNA isolation kit (Qiagen) according to the manufacturer's instructions. Amplification of DNA and RNA through PCR and RT-PCR model. Expression levels were normalized to the expression of the housekeeping gene $\beta$-actin using these primers: sense $5^{\prime}$-CGGGAAATCGTGCGTGACATT-3' and antisense 5'-GAGTT-
GAAGGTAGTTTCGTG-3', a 232-bp product. In order to evaluate $\mathrm{Bcl}-2$ is used to determine relevant changes of expression under some storage conditions such as cellular stress to synthesize these primers: sense $5^{\prime}$-TCCGCATCAGGAAGGCTAGA-3' and antisense 5'-AGGACCAGGCCTCCAAGC-3', a 113-bp product. The Bcl-2 gene was revealed by Western blot ( $\alpha$-actin and Bcl-2 antibody for total protein integrity).

\section{Conclusions}

With the advent of novel technologies, as well as the need to generate basic knowledge for the individualized management of patients with cancer, the creation of tumor tissue conservation banks has emerged. The role of the pathologist in the diagnosis and evaluation of the biological behavior of the neoplasms is crucial, and makes sure that the scientific process has repercussions in the best clinical practice as regards incorporating basic knowledge and clinical application through a tumor tissue bank that allows to do translational medicine. Therefore, the creation of a tumor tissue bank makes it possible to have sources available for the creation of new diagnostic biomarkers and to obtain therapeutic targets, which could exert a greater impact through subclassification of neoplastic diseases. In turn, this will lead to an advancement in legislative matters of tissue management, quality control methods, the participation of the patient in decision-making that is initiated with a letter of informed consent, and the fact that the scientific information obtained has repercussions on good medical practice. Finally, one crucial element of interinstitutional interest will be the transfer of these concepts to the different oncological centers, integrating in this manner a network that allows exploration of the different pathologies from therapeutic, epidemiological, and molecular points of view.

\section{References}

Organization of a Tumor Bank
Pathobiology 2010;77:147-154 
$>5$ Chung C, Wong S, Kian K, Hammond E, Dicker A, Harari P, Quinh-Thu L: Strategic plans to promote head and neck cancer translational research within the Radiation Therapy Oncology Group: a report from the Translational Research Program. Int J Radiat Oncol Biol Phys 2007;69:S67-S78.

$\checkmark 6$ Leyland-Jones B, Ambrosone C, Bartlett J, Ellis M, Enos R, Raji A, Pins M, Zujewski A, Hewitt SM, Forbes J, Abramovitz M, Braga S, Cardoso F, Harbeck N, Denkert C, Jewell S: Recommendations for collection and handling of specimens from group breast cancer clinical trials. J Clin Oncol 2008;26:56385644.

7 Hwang R, Wang H, Lara A, Gomez H, Chang T, Sieffert N, Moon Y, Ram S, Zimmerman $\mathrm{S}$, Lee J, Pisters P, Tamm E, Fleming J, Abbruzzese J, Evans D: Development of an integrated biospecimen bank and multidisciplinary clinical database for pancreatic cancer. Ann Surg Oncol 2008;15:13561366.

$>8$ Isabelle M, Teodorovic I, Morente M, Jamine D, Passioukov A, Lejeune S, Therasse P, Dinjens W, Oosterhuis J, Lam K, Oomen M, Spatz A, Racliffe C, Knox K, Mager R, Kerr $D$, Pezzella F, van de Vijver $M$, van Boven $H$, Alonso S, Kerjaschki D, Pammer J, LopezGuerrero J, Llombart Bosh A, Carbone A, Gloghini A, van Veen E, van Dame B, Riegman P: TuBaFrost 5: multifunctional central database application for a European tumor bank. Eur J Cancer 2006;42:3103-3109.

9 The Cooperative Human Tissue Network. http://chtn.nci.nih.gov/.
10 Morente M, de Alva E, Fernandez P: Tumour banking: the Spanish design. Pathobiology 2007;74:245-250.

11 http://housekeeping.about.com/od/vacuumcleaners/f/NeedaHEPA.htm.

12 http://www.cofepris.gob.mx/.

13 Rodrigues M, Vito I, Santos R, Paiva J, Pontes P, Silva P, Carneiro F: Establishment of a tumour bank: the experience of the Department of Pathology of Hospital S. João (Porto, Portugal). Cell Tissue Bank 2009; 10:75-77.

14 Suh KS, Remache YK, Patel JS, Chen SH, Haystrand R, Ford P, Shaikh AM, Wang J, Goy AH: Informatics-guided procurement of patient samples for biomarker discovery projects in cancer research. Cell Tissue Bank 2009; 10:43-48.

15 Knoppers BM: Biobanking: international norms. J Law Med Ethics 2005;33:7-14.

16 Knoppers BM: The babel of genetic data terminology. Nat Biotechnol 2005;23:925-927.

17 Hansson MG: Ethics and biobanks. Br J Cancer 2009; $100: 8-12$.

18 Riegman P, Morente M, Betsou F, de Blasio P, Geary P, Marble Arch International Working Group on Biobanking for Biomedical Research: Biobanking for better healthcare. Mol Oncol 2008;2:213-222.

19 Salvaterra E, Lecchi L, Giovanelli S, Butti B, Bardella MT, Bertazzi PA, Bosari S, Coggi G, Coviello DA, Lalatta F, Moggio M, Nosotti M, Zanella A, Rebulla P: Banking together. A unified model of informed consent for biobanking. EMBO Rep 2008;9:307-313.
20 Lunshof JE, Chadwick R, Vorhaus BD, Church MG: From genetic privacy to open consent. Nat Rev Genet 2008;9:407-409.

-21 Helgesson G, Dillner J, Carlson J, Bartram CR, Hansson MG: Ethical framework for previously collected biobank samples. Nat Biotechnol 2007;25:973-976.

22 Hewitt R, Watson P, Dhir R, Aamodt R, Thomas G, Mercola D, Grizzle W, Morente M: Timing of consent for the research use of surgically removed tissue. Is postoperative consenting acceptable? Cancer 2009;115:49.

23 http://www.capca.ca/english.asp?PageI.

24 Cambon-Thomsen A, Rial-Sebbag E, Knoppers BM: Trends in ethical and legal frameworks for the use of human biobanks. Eur Respir J 2007;30:373-382.

25 Chokshi DA, Parker M, Kwiatkowski DP: Data sharing and intellectual property in a genomic epidemiology network: policies for large-scale research collaboration. Bull World Health Org 2006;84:382-387.

26 Oosterhuis JW, Coebergh JW, van Veen EB: Tumour banks: well-guarded treasures in the interest of patients. Nat Rev Cancer 2003; 3:73-77.

27 http://conventions.coe.int/Treaty/EN/CadreListeTraites.

28 http://www.amaassn.org/apps/pf_online/ pf_online?f_n=browse\&doc=policyfiles / CEJA/E 8.HTM\&\&s_t $=\& s_{\text {t_p }} \mathrm{p}=\& \mathrm{nth}=$ $1 \&$ prev_pol=policyfiles/CEJA/E-1.02. HTM\&nxt_pol=policyfiles/CEJA/E-2.01. HTM\&> 